RI-08-01

hep-th/0108142

\title{
D-Branes on a gauged WZW model
}

\author{
Shmuel Elitzur and Gor Sarkissian \\ Racah Institute of Physics, The Hebrew University \\ Jerusalem 91904, Israel
}

The algebraic classification of Cardy for boundary states on a $G / H$ coset CFT of a compact group $\mathrm{G}$, is geometrically realized on the corresponding manifold resulting from gauging the WZW model. The branes consist of $\mathrm{H}$ orbits of quantized $\mathrm{G}$ conjugacy classes shifted by quantized $\mathrm{H}$ conjugacy classes.

$8 / 01$ 


\section{Introduction}

On a background of conformal field theory branes can be described by boundary states. Demanding preservation of maximal symmetry, these boundary states were classified by Cardy [1] for a rational CFT. In that case a boundary state, that is, a type of a D-brane, was found to exist in correspondence to each primary field of the chiral algebra of the given CFT. Geometrically D-branes are embedded as subsets of target space. Whenever a CFT target space possesses a geometrical interpretation the algebraically constructed Cardy boundary states should be realized as such subsets. In the case of a CFT which is a compact group manifold such a realization exists [2] , [3, 4, 5, 5, 6, 0, 8, 9, 10, 11, 12, 13, 14].

D-branes can sit on a finite set of allowed conjugacy classes on the group manifold which are in a natural correspondence with the representations of the affine group generated from the primary fields present in the model.

In this work we study a geometrical realization of D branes for a more general class of conformal field theories, the coset models $G / H$ where $H$ is a subgroup of a compact group $G$. Algebraically this is a rational CFT whose allowed boundary states obey the Cardy's classification. A geometrical description of such models is provided when considered as gauged WZW models integrating out the gauge fields [15], [16], [17].

Following the method applied to CFT on a group manifold we construct a WZW action for a world sheet with boundary moving on the group manifold $G$ with the subgroup $H$ gauged away. The boundary is constrained into some subsets of $G$ dictated by the preserved symmetry. The consistency of this action puts some quantization conditions on the allowed positions of the branes, establishing their correspondence to the primary fields of the coset CFT. The dimensionalities of the resulting generic branes are determined.

We limit ourself to maximally symmetric branes. The more general case of branes with a smaller symmetry was studied for the parafermionic example in [18].

We deal with non-abelian $G$ and $H$. The case of $U(1)$ gauge group was discussed extensively recently, in particular with respect to the parafermionic example [18].

In sec. 2 the analysis of branes on a group manifold is reviewed. Sec. 3 deals with the $G / H$ model. In sec. 4 the case of $G$ and $H$ with a common center is discussed. In an appendix, some formulae from the main text are motivated.

After completing this work we found the paper [19] by K. Gawedzki, whose sec. 2 contains results similar to ours. 


\section{Branes on a WZW model}

As a preparation to the case of cosets we review here the case of a group manifold. We follow the discussion of [2], where the $\mathrm{SU}(2)$ case is dealt with and the generalization to any compact group in [5]. We check explicitely the symmetries of the resulting action.

The action of a WZW model for a world sheet $\Sigma$ without boundary is

$$
S=S^{\mathrm{kin}}+S^{\mathrm{WZ}}=\frac{k_{G}}{4 \pi}\left[\int_{\Sigma} d^{2} z L^{\mathrm{kin}}+\int_{B} \omega^{\mathrm{WZ}}\right]
$$

where $L^{\mathrm{kin}}=\operatorname{Tr}\left(\partial_{z} g \partial_{\bar{z}} g^{-1}\right), \omega^{\mathrm{WZ}}=\frac{1}{3} \operatorname{Tr}\left(g^{-1} d g\right)^{3}$, and $B$ is a three-manifold bounded by $\Sigma$. Here $g(z, \bar{z})$ is the embedding of $\Sigma$ into the compact group manifold $G$ and the integer $k_{G}$ is the level of the model. This action is invariant under the $\hat{G} \times \hat{G}$ symmetry, $\hat{G}$ being the loop group of $G$,

$$
g(z, \bar{z}) \rightarrow h_{L}(z) g(z, \bar{z}) h_{R}(\bar{z})
$$

This invariance can be seen by substituting $h_{L}(z) g(z, \bar{z}) h_{R}(\bar{z})$ for $g$ in (2.1) using the Polyakov - Wiegmann identities [20], [21]:

$$
\begin{gathered}
L^{\mathrm{kin}}(g h)=L^{\mathrm{kin}}(g)+L^{\mathrm{kin}}(h)-\left(\operatorname{Tr}\left(g^{-1} \partial_{z} g \partial_{\bar{z}} h h^{-1}+\operatorname{Tr}\left(g^{-1} \partial_{\bar{z}} g \partial_{z} h h^{-1}\right)\right)\right. \\
\omega^{\mathrm{WZ}}(g h)=\omega^{\mathrm{WZ}}(g)+\omega^{\mathrm{WZ}}(h)-d\left(\operatorname{Tr}\left(g^{-1} d g d h h^{-1}\right)\right)
\end{gathered}
$$

together with the fact that $\partial_{z} h_{R}=\partial_{\bar{z}} h_{L}=0$.

When $\Sigma$ has boundaries, left moving and right moving waves are mixed by them. The two independent symmetries in (2.2) cannot be present. Still one can put boundary conditions which preserve the symmetry (2.2) in the bulk with $h_{L}$ and $h_{R}^{-1}$ tending on the boundary to a common limit. The symmetry on the boundary is then

$$
g \rightarrow h(\tau) g h(\tau)^{-1}
$$

where $\tau$ parameterizes the boundary. The boundary conditions on $g$ should respect the symmetry (2.5) . If a group element $f$ is allowed on the boundary, the symmetry implies that $k f k^{-1}$ should also be allowed, for any $k \in G$. Thus the boundary should be allowed to take values in the whole conjugacy class $C_{f}^{G}$ defined by

$$
C_{f}^{G}=\left\{g \in G \mid g=k f k^{-1}\right\}
$$


Suppose then that the boundary of $\Sigma$ is constrained to map into the conjugacy class $C_{f}^{G}$

$$
g(\tau)=k(\tau) f k^{-1}(\tau)
$$

for some fixed group element $f$. As it stands the action (2.1) is not well defined for a world sheet with boundary. There is no region $B$ bounded by $\Sigma$ when $\Sigma$ itself has boundary. To fix that, for a world sheet with a single hole, one extends the mapping from $\Sigma$ to $G$ to a surface without boundary $\Sigma \cup D$ where $D$ is an auxiliary disc which closes the hole in $\Sigma$ having a common boundary with it [2]. The disc $D$ is mapped into the same conjugacy class (2.7) allowed for its boundary. The region $B$ in the action (2.1) is then taken to be bounded by $\Sigma \cup D$. The action should be formulated such that the embedding of the interior of the auxiliary disc $D$ inside the conjugacy class does not matter as long as its boundaries are fixed. We also demand that the symmetry (2.5) which in general does affect also the boundaries of $D$ will continue to be respected by the action. This is done by modifying the action into:

$$
S=\frac{k_{G}}{4 \pi}\left[\int_{\Sigma} d^{2} z L^{\mathrm{kin}}+\int_{B} \omega^{\mathrm{WZ}}-\int_{D} \omega^{f}(k)\right]
$$

where $\partial B=\Sigma \cup D$ and $\omega^{f}(k)$ is the two-form defined on the conjugacy class (2.7) for $g=k f k^{-1}$ as [2],

$$
\omega^{f}(k)=\operatorname{Tr}\left(k^{-1} d k f k^{-1} d k f^{-1}\right)
$$

On the class (2.7), where it is defined, $\omega^{f}$ satisfies

$$
d \omega^{f}=\omega^{\mathrm{WZ}}
$$

Under the transformation (2.2) with boundary values (2.5), the change in the $L^{\text {kin }}$ term in (2.8) read from (2.3), is canceled by the corresponding $\Sigma$ integral of the boundary term from the change in the $\omega^{\mathrm{WZ}}$ term, read from (2.4). In the presence of a world sheet boundary there remains the contribution from $D$ to the latter change,

$$
\Delta\left(S^{\mathrm{kin}}+S^{\mathrm{WZ}}\right)=\frac{k}{4 \pi} \int_{D} \operatorname{Tr}\left[h^{-1} d h\left(g h^{-1} d h g^{-1}-g^{-1} d g-d g g^{-1}\right)\right]
$$

The change in the $\omega^{f}$ term in $(2.8)$ can be read from the identity

$$
\omega^{f}(h k)-\omega^{f}(k)=\operatorname{Tr}\left[h^{-1} d h\left(g h^{-1} d h g^{-1}-g^{-1} d g-d g g^{-1}\right)\right]
$$


where $g=k f k^{-1}$, which follows from the definition (2.9) . The change in the $\int_{D} \omega^{f}(k)$ term exactly cancels (2.11) so that (2.8) is indeed invariant under (2.2) .

Eq. 2.10) guarantees that the action is invariant under continuous deformations of the embedding of the auxiliary disc inside the conjugacy class. The change in the $\omega^{f}$ integral cancels the integral of the $\omega^{\mathrm{WZ}}$ term on the three-volume swept by the disc during such a deformation. Since in general the second homotopy of a conjugacy class is non trivial, there are different embeddings of a disc in such a class which are not continuously connected. The union of two such embedded discs is not the boundary of a three volume inside the conjugacy class, where (2.10) is valid. The action (2.8) is in fact sensitive to such a topological change in the embedding of the auxiliary disc. In order that this change will have no physical effect, the induced change in the action must be an integral multiple of $2 \pi$. This leads [2], [5] to a quantization of the conjugacy classes allowed for as boundary conditions. Since $k$ in $(2.7)$ is defined modulo right multiplication by any element commuting with $f$ and the group of such elements for a generic $f$ is isomorphic to $T^{G}$, the Cartan torus of $G$, the conjugacy class (2.6) can be described as $G / T^{G}$. Its second homotopy group is therefore,

$$
\Pi^{2}\left(C_{f}^{G}\right)=\Pi^{1}\left(T^{G}\right)
$$

If $r$ is the rank of $G$, a topologically non trivial embedding of $S^{2}$ in $C_{f}^{G}$ is characterized by an $r$ dimensional vector in the coroot lattice of $G$. Namely, if one embedding $D$ of the disc into $C_{f}^{G}$ is given by $k f k^{-1}$ and another embedding $D^{\prime}$ sends it into $k^{\prime} f k^{\prime-1}$, then on the topologically circular boundary the two embeddings should coincide. This implies

$$
k(\tau) k^{\prime}(\tau)^{-1}=t(\tau)
$$

where $t(\tau)$ is an element of the subgroup isomorphic to $T^{G}$ which commutes with $f$. Eq. (2.14) determines a mapping from the circular boundary of a given hole in the world sheet into the torus $T^{G}$. Since $T^{G}$ is $R^{r}$ modulo $2 \pi$ times the coroot lattice, every such mapping belongs to a topological sector parameterized by a vector in the coroot lattice describing the winding of this circle on the torus $T^{G}$. This lattice vector determines, by $(2.13)$, the element of $\Pi^{2}\left(C_{f}^{G}\right)$ corresponding to the union of $D$ and $D^{\prime}$.

Let the element $\mathrm{f}$ in (2.7) chosen in the Cartan torus be of the form $f=e^{i \theta \cdot \lambda}$ where $\lambda$ are Cartan generators. The change in the action resulting from a topological change in 
the embedding of the disc which is characterized by a coroot lattice vector $s$, is given by [5]

$$
\Delta S=k_{G}(\theta \cdot s)
$$

where the length of long roots is normalized to 2 . Consistency of the model then implies the condition

$$
\theta \cdot \alpha \in 2 \pi Z / k_{G}
$$

for any coroot $\alpha$. In this normalization the weight lattice is the set of points in $R^{r}$ whose scalar product with any coroot takes integral values. Eq. 2.16 implies then, that $\theta$ should be $2 \pi / k_{G}$ times a vector in the weight lattice. As a point in $T^{G}, \theta$ is defined modulo $2 \pi$ times the coroot lattice. The allowed conjugacy classes correspond then to points in the weight lattice divided by $k_{G}$, modulo the coroot lattice. This is also the characterization of the integrable representations of $\hat{G}$, the affine $G$ algebra at level $k_{G}$, which correspond to the primary fields of the WZW model. It is in accordance with the algebraic analysis of Cardy [1] where a correspondence is established between primary operators in a rational CFT and boundary states. The above discussion is a geometrical realization on the group manifold of this correspondence.

It is of course equally consistent and symmetric to replace the set of conjugacy classes (2.7) with quantization condition (2.16), by the same classes shifted by a fixed group element $m$. The boundary conditions $(2.7)$ are then modified into

$$
g(\tau)=k(\tau) f k^{-1}(\tau) m
$$

where $\mathrm{f}$ satisfies (2.16) and $\mathrm{m}$ is an arbitrary fixed group element. This amounts to constrain the boundary values of $g$ into the shifted conjugacy class $C_{f}^{G} m$. This set of boundary conditions preserves a boundary symmetry different from (2.5) . The shifted symmetry is

$$
g \rightarrow h(\tau) g m^{-1} h(\tau)^{-1} m
$$

If we insist on the boundary symmetry (2.5), the allowed boundary states are the set (2.7) with the condition (2.16). 


\section{Branes on a Coset}

Let $G$ be a compact, simply connected, non-abelian group. The $G / H$ coset CFT, where $H$ is a subgroup of $G$, can be described in terms of a gauged WZ action [15], where the symmetry

$$
g \rightarrow h g h^{-1}
$$

$g \in G, h \in H$ is gauged away. An $H$ Lie algebra valued world sheet vector field $A$ is added to the system, and the $G / H$ action on a world sheet without boundary becomes,

$$
\begin{aligned}
& S^{G / H}=S^{\mathrm{kin}}+S^{\mathrm{WZ}}+S^{\text {gauge }} \\
& =\frac{k_{G}}{4 \pi}\left[\int_{\Sigma} d^{2} z L^{\mathrm{kin}}+\int_{B} \omega^{\mathrm{WZ}}\right] \\
& +\frac{k_{G}}{2 \pi} \int_{\Sigma} d^{2} z \operatorname{Tr}\left(A_{\bar{z}} \partial_{z} g g^{-1}-A_{z} g^{-1} \partial_{\bar{z}} g+A_{\bar{z}} g A_{z} g^{-1}-A_{z} A_{\bar{z}}\right)
\end{aligned}
$$

Introduce $H$ group element valued world sheet fields $U$ and $\tilde{U}$ as [22], [23],

$$
\begin{aligned}
& A_{z}=\partial_{z} U U^{-1} \\
& A_{\bar{z}}=\partial_{\bar{z}} \tilde{U} \tilde{U}^{-1}
\end{aligned}
$$

Denote the action (2.1) by $S^{G}(g)$. Then the coset action (3.2) becomes,

$$
S^{G / H}=S^{G}\left(U^{-1} g \tilde{U}\right)-S^{H}\left(U^{-1} \tilde{U}\right)
$$

as can be checked using Polyakov Wiegmann identities (2.3), (2.4) and the definitions (3.3) . The level $k_{H}$ of the $S^{H}$ term in (3.4), is related to $k_{G}$ through the embedding index of $H$ in $G$ [24].

The model has then the following symmetries. First of all one should identify configurations related by the local gauge transformation,

$$
\begin{aligned}
g(z, \bar{z}) & \rightarrow h(z, \bar{z}) g(z, \bar{z}) h^{-1}(z, \bar{z}) \\
U(z, \bar{z}) & \rightarrow h(z, \bar{z}) U(z, \bar{z}) \\
\tilde{U}(z, \bar{z}) & \rightarrow h(z, \bar{z}) \tilde{U}(z, \bar{z})
\end{aligned}
$$

with $h(z, \bar{z}) \in H$. In addition, by (2.2), there are further global symmetries

$$
\begin{aligned}
U(z, \bar{z}) & \rightarrow U(z, \bar{z}) h_{L}^{-1}(z) \\
\tilde{U}(z, \bar{z}) & \rightarrow \tilde{U}(z, \bar{z}) h_{R}(\bar{z}) \\
g(z, \bar{z}) & \rightarrow g(z, \bar{z})
\end{aligned}
$$


where $h_{L}, h_{R} \in H$. The action is also invariant under

$$
\begin{aligned}
g(z, \bar{z}) & \rightarrow m_{L}(z) g(z, \bar{z}) m_{R}(\bar{z}) \\
U & \rightarrow U \\
\tilde{U} & \rightarrow \tilde{U}
\end{aligned}
$$

with $m_{L}, m_{R} \in G$ and $\left[m_{L}, H\right]=\left[m_{R}, H\right]=0$.

Suppose now that we gauge the WZW model of the group $G$, defined on a world sheet with boundary with the boundary conditions 2.7 on $g(z, \bar{z})$. On the group manifold, we saw in previous section that boundary conditions corresponding to shifted conjugacy classes (2.17) did not preserve the same symmetry of the condition (2.7) but rather a different symmetry (2.18) . Insisting on the symmetry (2.2) we did not include this kind of conditions. Here on the coset, the symmetry (3.7) is limited relative to (2.2), and we note that shifted boundary conditions like (2.17) are still consistent with them provided the shift element $m$ in (2.17) belongs to $H$. We will then consider a more general type of boundary conditions on $g$ allowing on the boundary

$$
g(\tau)=k(\tau) f k^{-1}(\tau) l
$$

with $f, k \in G$ and $l$ an element in $H$. For a fixed $l$, these boundary conditions are not gauge invariant. The gauge symmetry (3.5) forces us to allow, together with shifting by $l$, to shift by any element in its $H$ conjugacy class. We are then lead to the following boundary conditions,

$$
g(\tau)=k(\tau) f k^{-1}(\tau) p(\tau) l p^{-1}(\tau)
$$

with $p(\tau), l \in H$. In other words, on the boundary $g$ is constrained to a product $C_{f}^{G} C_{l}^{H}$ of a $G$ conjugacy class with an $H$ class.

As in previous section the presence of the WZ term necessitates an auxiliary disc $D$ for each hole in the world sheet, to define the boundary of the three-volume integral in the action. To proceed, we will continue from the boundary into the disc $D$ the field $g$ subject to conditions (3.9). Here again an additional two-form has to be added on the disc to give meaning to the action. For the product of classes boundary conditions (3.9) the two-form introduced in (2.9) is not appropriate. To complete the action define another two-form as follows. Let $c_{1}=k f k^{-1}, f \in G$, and $c_{2}=p l p^{-1}, l \in H$. Define

$$
\Omega^{(f, l)}(k, p)=\omega^{f}(k)+\operatorname{Tr}\left(d c_{2} c_{2}^{-1} c_{1}^{-1} d c_{1}\right)+\omega^{l}(p)
$$


where $\omega^{f}(k)$ and $\omega^{l}(p)$ are two-forms defined as in (2.9). Considerations leading to this particular form are discussed in the appendix. We will then add to the action (3.2) the term

$$
-\frac{k_{G}}{4 \pi} \int_{D} \Omega^{(f, l)}(k, p)
$$

To check the consistency of the action with the term (3.11), notice first that for $g=c_{1} c_{2}$, the WZ term has the form

$$
\omega^{\mathrm{WZ}}=\frac{1}{3} \operatorname{Tr}\left(c_{1}^{-1} d c_{1}\right)^{3}+\frac{1}{3} \operatorname{Tr}\left(c_{2}^{-1} d c_{2}\right)^{3}+\operatorname{Tr}\left(c_{1}^{-1} d c_{1}\right)^{2} d c_{2} c_{2}^{-1}+\operatorname{Tr}\left(c_{1}^{-1} d c_{1}\right)\left(d c_{2} c_{2}^{-1}\right)^{2}
$$

Using 2.10 one gets,

$$
d \Omega^{(f, l)}=\omega^{\mathrm{WZ}}
$$

This guarantees the invariance of the action (3.2) with the additional term (3.11) under a continuous deformation of the embedding of the disc $D$ into $G$ subject to conditions (3.9).

As to the symmetry (3.7) with $m_{L}=m_{R}^{-1}=m$ on the boundary, it acts on the term (3.11) taking $c_{1} \rightarrow m c_{1} m^{-1}$ for $[m, H]=0$. The change in $\Omega^{(f, l)}$ is given by

$$
\Delta \Omega^{(f, l)}=\operatorname{Tr}\left[\left(m^{-1} d m\right)\left(c_{1} m^{-1} d m c_{1}^{-1}-d c_{1} c_{1}^{-1}-c_{1}^{-1} d c_{1}-d c_{2} c_{2}^{-1}-c_{1} d c_{2} c_{2}^{-1} c_{1}^{-1}\right)\right]
$$

This exactly cancels the variation of the bulk terms read from (2.11) when $m$ is substituted there for $h, c_{1} c_{2}$ for $g$ and the commutation of $m$ with $c_{2}$ taken into account.

As in previous section, the embedding of the disc $D$ into $C_{f}^{G} C_{l}^{H}$ involves a topological choice. Holding $p l p^{-1}$ in (3.9) fixed on the disc while performing a topological change corresponding to a $G$ coroot lattice vector $s_{G}$ in the definition on the interior of $D$, of the factor $k f k^{-1}$, will induce in $S$ in (3.2) the same change as that of previous section

$$
\Delta_{G} S=k_{G}\left(\theta_{G} \cdot s_{G}\right)
$$

where $f=e^{i \theta_{G} \cdot \lambda_{G}}$. The consistency of the action requires then the same quantization condition (2.16) on the $G$ conjugacy class

$$
\theta_{G} \cdot \alpha_{G} \in 2 \pi Z / k_{G}
$$

Similarly, a topological change corresponding to an $H$ coroot vector $s_{H}$ in the continuation to $D$ of the factor $p l p^{-1}$ in (3.9) with the $k f k^{-1}$ held fixed, will also change $S$. For $l=e^{i \theta_{H} \lambda_{H}}$ this change will be

$$
\Delta_{H} S=k_{H}\left(\theta_{H} \cdot s_{H}\right)
$$


The consistency of the action (3.4) then also constrains the $H$ conjugacy class factor by

$$
\theta_{H} \cdot \alpha_{H} \in 2 \pi Z / k_{H}
$$

There is a problem in the two-form $\Omega^{(f, l)}(k, p)$ introduced into the action in eq. (3.11). This form depends explicitly on the $G$ and $H$ classes factors of the group element $g$ on the boundary and in the disc. In general, however, the factorization of an element $g \in C_{f}^{G} C_{l}^{H}$ into a product of the form $g=k f k^{-1} p l p^{-1}$ is not unique. Let $c_{1}=k f k^{-1}$ and $c_{2}=p l p^{-1}$ . Varying $k$ infinitesimally by

$$
\delta k=q_{G} k
$$

and $p$ by

$$
\delta p=q_{H} p
$$

where $q_{G}$ and $q_{H}$ belong to $G$ and $H$ Lie algebras respectively, $c_{1}$ is changed by $\delta c_{1}=$ $\left[q_{G}, c_{1}\right]$ and $c_{2}$ varies by $\delta c_{2}=\left[q_{H}, c_{2}\right]$. If the Lie algebra elements $q_{G}$ and $q_{H}$ are chosen such that they satisfy

$$
q_{G}-c_{1}^{-1} q_{G} c_{1}=q_{H}-c_{2} q_{H} c_{2}^{-1}
$$

then the product $g=c_{1} c_{2}$ is unchanged under the above variation. The left hand side of eq. (3.21) is a linear operator in the Lie algebra of $G$ acting on $q_{G}$. This operator has a null space of dimension $r_{G}$, the rank of $G$, namely the $r_{G}$ independent elements commuting with $c_{1}$. Choosing $c_{1}$ in the Cartan torus of $G$ this null space is the Cartan subalgebra of $G$. For a $q_{H}$ in the $H$ Lie algebra, which does not commute with $c_{2}$, there will be a $q_{G}$ solving (3.21) provided that $q_{H}-c_{2} q_{H} c_{2}^{-1}$ has no component in this Cartan algebra. The dimension of the set of pairs $\left(q_{G}, q_{H}\right)$ solving (3.21), is the dimension of the set of elements $q_{H}$ not counting their components along the Cartan algebra commuting with $c_{2}$, such that $q_{H}-c_{2} q_{H} c_{2}^{-1}$ has no components in the algebra commuting with $c_{1}$. This is the dimension of different factorizations of a given $g=c_{1} c_{2}$ in $C_{f}^{G} C_{l}^{H}$ as a product of a pair of elements from both classes. For generic $c_{1}$ and $c_{2}$, for which the two Cartan algebras commuting with $c_{1}$ and $c_{2}$ are disjoint, this dimension is $d_{H}-2 r_{H}$. At a generic $g=c_{1} c_{2}$ point in the region $C_{f}^{G} C_{l}^{H}$ its dimension is then given by

$$
d\left(C_{f}^{G} C_{l}^{H}\right)=d_{G}-r_{G}+d_{H}-r_{H}-\left(d_{H}-2 r_{H}\right)=d_{G}-r_{G}+r_{H}
$$

Non generic points for which the algebra commuting with $c_{1}$ and that commuting with $c_{2}$ have a common subspace, form lower dimensional boundary in $G$ of the region $C_{f}^{G} C_{l}^{H}$. 
Since fixing $g \in C_{f}^{G} C_{l}^{H}$ does not determine $c_{1}$ and $c_{2}$ the two-form $\Omega$ in the action (3.11) seems to require more data than just $g$. In fact one can check the behavior of $\Omega^{(f, l)}(k, p)$ under the variation (3.19) and (3.20) and find that it is not invariant even when $q_{G}$ and $q_{H}$ do satisfy (3.21) . Explicitly, in this case we find

$$
\delta \Omega^{(f, l)}(k, p)=-2 d\left[\operatorname{Tr}\left(q_{G} d c_{1} c_{1}^{-1}+q_{H} c_{2}^{-1} d c_{2}\right)\right]
$$

It seems then that for the action (3.11) to make sense, one should allow for extra degrees of freedom on the boundary, fixing the factorization of $g$ there as a product of $G$ and $H$ conjugacy classes. Namely, the Physics does depend on $k$ and $p$ in (3.9), not just on the value of $g$. Note though, that there is no dependence on the factorization chosen inside the disc, once it is fixed on the boundary. Since the variation of $\Omega^{(f, l)}$ in (3.23) has the form of a derivative of a local one form, as could be expected from (3.13), the variation vanishes for $q_{G}$ and $q_{H}$ zero on the boundaryt.

The allowed D branes for the $G / H$ model correspond then to a pair of quantized conjugacy classes of the two groups. Since each such a class corresponds to an integrable representation, we get a characterization of these branes by pairs of a $G$ primary field and an $H$ primary field. This is again in accordance with Cardy's analysis, the primary fields of the $G / H$ CFT, generically correspond to such pairs.

A geometric picture for the $G / H$ coset model emerges once the gauge is fixed and the gauge fields are integrated over [15]. The points of the resulting manifold can be identified as the orbits in $G$ of the gauge transformation (3.1) . The boundary conditions (3.9) put the branes on $G$ conjugacy classes in the $G$ group manifold, quantized according to (3.16), shifted, in the sense of (2.17), by $H$ conjugacy classes, which are also quantized according to (3.18) . All the points on the orbit of a gauge transformation (3.1) are identified. For generic $f \in G$ and $l \in H$ the dimension of the product $C_{f}^{G} C_{l}^{H}$ at a generic point is given in eq. (3.22) . Identifying the $H$ gauge orbits generically reduces the dimension by $d_{H}$. The generic dimension of the geometrically realized $D$ branes is then, for non abelian $G$ and $H$,

$$
\operatorname{dim}(\text { brane })=d_{G}-d_{H}-r_{G}+r_{H} .
$$

1 See [19] for a discussion of this issue in the framework of the canonical formalism. 


\section{The case of a common center}

When $H$ contains some subgroup $C$ of the center of $G$, the above discussion gets modified in two ways. First, for $z \in C$ the region $C_{f}^{G} C_{l}^{H}$ is identical to the region $C_{z f}^{G} C_{z^{-1} l}^{H}$. The brane corresponding to the pair $(f, l)$ of conjugacy classes is then identical to the brane corresponding to the pair $\left(z f, z^{-1} l\right)$. This is the geometrical origin of the phenomena known in the context of coset CFT without boundary as "field identification" [25], [26], [24]. It is again consistent with Cardy's identification of boundary states with primary fields.

Since the gauge transformation takes $g$ into $h g h^{-1}$, it does not distinguish between the transformations $h$ and $z h$ for any $z \in C$. We can then think of the gauge group as $H / C$. Recall that the element $k$ in (3.9) is defined modulo right multiplication by $G$ group elements from the torus $T_{f}^{G}$ commuting with $f$. Similarly $p$ in that equation can be multiplied from the right by any element of $T_{l}^{H}$. Let the boundary of the hole be parameterized by $0 \leq \tau \leq 2 \pi$. We have seen in (2.14) that upon replacing the boundary value $k(\tau)$ by

$$
k^{\prime}(\tau)=k(\tau) t(\tau)
$$

with

$$
t(\tau)=e^{\frac{i}{2 \pi} \tau(s \cdot \lambda)}
$$

$s$ being a coroot lattice vector and $\lambda$ a vector of generators commuting with $f$, continuing $k^{\prime}$ rather than $k$ into the disc, the change (3.15) is induced in the action. This gave rise to the quantization condition (3.16). A similar independent change in $p(\tau)$ induces the change (3.17) leading to the condition (3.18). Recall also that a gauge transformation $h \in H$ multiplies both $k$ and $p$ by $h$ from the left. Let $z \in C$ be represented as

$$
z=e^{i(w \cdot \lambda)} .
$$

Notice that $w$ is a common weight vector of $G$ and $H$. Consider an $H / C$ gauge transformation $h(z, \bar{z}) \in H$, which satisfies on the boundary of the hole

$$
h(0)=z^{-1} h(2 \pi)
$$

Let this transformation act on a configuration with a given continuous choice of $k$ and $p$ on the boundary and inside the disc. On the world sheet $\Sigma$ the action density, being gauge 
invariant, does not change. The representation (3.9) of $g$ on the boundary is changed, the transformed $k$ and $p$ satisfy

$$
\begin{aligned}
& k(0)=z^{-1} k(2 \pi) \\
& p(0)=z^{-1} p(2 \pi)
\end{aligned}
$$

In this form $k$ and $p$ are discontiuous in $H$. They are continuous in $H / C$, but the paths $k(\tau)$ and $p(\tau)$ of (4.5) are non contractible in $H / C$ and cannot be continued into the interior of the disc to be substituted in the action (3.11). To define the action we must, before continuing into the disc, to redefine $k$ and $p$ according to (4.1), multiplying them from the right by an appropriate Cartan element, changing $k$ into $k^{\prime}$ and $p$ into $p^{\prime}$ defined as

$$
\begin{aligned}
& k^{\prime}(\tau)=k^{\prime}(\tau) e^{\frac{i}{2 \pi} \tau(w \cdot \lambda)} \\
& p^{\prime}(\tau)=p^{\prime}(\tau) e^{\frac{i}{2 \pi} \tau(w \cdot \lambda)}
\end{aligned}
$$

The redefined $k^{\prime}$ and $p^{\prime}$ are contractible and can be continued into the disc. The redefinition (4.6), like (4.1), induces a change in the disc term of the action, according to (3.15) and (3.17). Notice that, unlike (4.2), (4.6) contains a weight vector rather than a root vector, and that this twist is done together on $k$ and on $p$. Equations (3.15) and (3.17) give then for the change of the action induced by (4.6)

$$
\Delta S=\left(k_{G} \theta_{G}+k_{H} \theta_{H}\right) \cdot w
$$

where $f=e^{i\left(\theta_{G} \cdot \lambda\right)}$ and $l=e^{i\left(\theta_{H} \cdot \lambda\right)}$. Invariance under the gauge transformation (4.4) requires this change to be a multiple of $2 \pi$ leading to a further condition, a correlation between $G$ and $H$ conjugacy classes,

$$
\left(k_{G} \theta_{G}+k_{H} \theta_{H}\right) \cdot w \in 2 \pi Z
$$

for every common weight of $G$ and $H$. This is again in accordance with Cardy's correspondence of boundary states with primary fields of the CFT without boundary. The condition (4.8) for coset CFT is known as the selection rule [25], 26], [24], demanding the same behavior of members of the pair of $G$ and $H$ representations under the common center. 
Acknowledgments: We thank M. Berkooz, A. Giveon, D. Kazhdan, D. Kutasov, E. Rabinovici, N. Seiberg and A. Schwarz for useful discussions. This work is supported in part by the Israel Academy of Sciences and Humanities - Centers of Excellence Program.

\section{Appendix A.}

Here we present an alternative reasoning bringing to the boundary condition (3.9) and the two-form (3.10).

Let us study the action (3.2) in the presence of boundary with the boundary condition (2.7) modified with boundary term $\int_{D} \omega^{f}(k)$ :

$$
\begin{aligned}
& S=\frac{k_{G}}{4 \pi}\left[\int_{\Sigma} d^{2} z L^{\mathrm{kin}}+\int_{B} \omega^{\mathrm{wzw}}-\int_{D} \omega^{f}(k)\right] \\
& +\frac{k 2}{\pi} \int_{\Sigma} d^{2} z \operatorname{Tr}\left(A_{\bar{z}} \partial_{z} g g^{-1}-A_{z} g^{-1} \partial_{\bar{z}} g+A_{\bar{z}} g A_{z} g^{-1}-A_{z} A_{\bar{z}}\right)
\end{aligned}
$$

where $\left.g\right|_{D}=c_{1}=k f k^{-1}$. Using again the parameterization (3.3), by straitforward computation we can show that

$$
\begin{aligned}
& S=S^{G}\left(U^{-1} g \tilde{U}\right)-S^{H}\left(U^{-1} \tilde{U}\right) \\
& -\int_{D}\left[\omega^{f}\left(U^{-1} k\right)+\operatorname{Tr}\left(d\left(U^{-1} \tilde{U}\right)\left(U^{-1} \tilde{U}\right)^{-1}\left(U^{-1} c_{1} U\right)^{-1} d\left(U^{-1} c_{1} U\right)\right)\right]
\end{aligned}
$$

Till now we did not specify boundary conditions for the gauge field. Denote $U^{-1} \tilde{U}=h$. We demand that at the boundary

$$
h=\left.U^{-1} \tilde{U}\right|_{D}=c_{2}=p l p^{-1}
$$

which is consistent with (3.5) and (3.6) for $h_{L}=h_{R}^{-1}$. Adding and subtracting to the action (A.2) the expression $\int_{D} \omega^{l}(p)$ we can write it in the form:

$$
\begin{aligned}
& S=S^{G}\left(U^{-1} g \tilde{U}\right)-\int_{D}\left[\omega^{f}\left(U^{-1} k\right)+\operatorname{Tr}\left(d c_{2} c_{2}^{-1}\left(U^{-1} c_{1} U\right)^{-1} d\left(U^{-1} c_{1} U\right)\right)+\omega^{l}(p)\right] \\
& -\left[S^{H}(h)-\int_{D} \omega^{l}(p)\right]
\end{aligned}
$$

Defining the two-form

$$
\Omega^{(f, l)}(k, p)=\omega^{f}(k)+\operatorname{Tr}\left(d c_{2} c_{2}^{-1} c_{1}^{-1} d c_{1}\right)+\omega^{l}(p)
$$


where as before $c_{1}=k f k^{-1}$ and $c_{2}=p l p^{-1}$, we can compactly re-write (A.4) as follows:

$$
S=S^{G}\left(U^{-1} g \tilde{U}\right)-\int_{D} \Omega^{(f, l)}\left(U^{-1} k, p\right)-\left[S^{H}(h)-\int_{D} \omega^{l}(p)\right]
$$

Note that the two-form (A.5) shows the same behavior under a shift of its arguments as that of $\omega^{f}$ in (2.12), namely

$$
\Omega^{(f, l)}(h k, h p)-\Omega^{(f, l)}(k, p)=\operatorname{Tr}\left[h^{-1} d h\left(g h^{-1} d h g^{-1}-g^{-1} d g-d g g^{-1}\right)\right],
$$

where $g=k f k^{-1} p l p^{-1}$. Finally rearranging terms in (A.6) and (A.1) we get

$$
\begin{aligned}
& {\left[S^{G}(g)-\int_{D} \omega^{f}(k)\right]+\left[S^{H}(h)-\int_{D} \omega^{l}(p)\right]=S^{G}\left(U^{-1} g \tilde{U}\right)-\int_{D} \Omega^{(f, l)}\left(U^{-1} k, p\right)} \\
& -\frac{k_{G}}{2 \pi} \int_{\Sigma} d^{2} z \operatorname{Tr}\left(A_{\bar{z}} \partial_{z} g g^{-1}-A_{z} g^{-1} \partial_{\bar{z}} g+A_{\bar{z}} g A_{z} g^{-1}-A_{z} A_{\bar{z}}\right)
\end{aligned}
$$

We see that the sum of the two actions of the WZW models with boundary can be derived after gauging the action given by the first two terms of the right hand side of (A.8). Let us study this action in more details. First of all we see that from the conditions that $g$ and $U^{-1} \tilde{U}$ lie at the boundary in the conjugacy classes $k f k^{-1}$ and $p l p^{-1}$ respectively, follows that argument of the action in r.h.s. of (A.8) $U^{-1} g \tilde{U}$ lies in the product of these conjugacy classes:

$$
\left.U^{-1} g \tilde{U}\right|_{D}=\left.U^{-1} g U U^{-1} \tilde{U}\right|_{D}=U^{-1} k f k^{-1} U p l p^{-1}=\left(U^{-1} k\right) f\left(U^{-1} k\right)^{-1} p l p^{-1}
$$

From the fact that the actions in the l.h.s. of (A.8) do not change under infinitesimal variations of the location of the auxiliary disc we expect that also the action on the r.h.s. does not change under them. i.e. as we explained in section $2 d \Omega^{f, l}=\omega^{\mathrm{WZ}}$ on the product of conjugacy classes, which was explicitly checked in section 3 . 


\section{References}

[1] J.L. Cardy, Nucl. Phys. B324 (1989) 581.

[2] A. Alekseev and V. Schomerus, hep-th/9812193.

[3] C. Klimcik and P. Severa, Nucl. Phys. B488 (1997) 653.

[4] S. Stanciu, hep-th/9901122, JHEP 9909 (1999) 028.

[5] K. Gawedzki, hep-th/9904145.

[6] L. Birke, J. Fuchs and C. Schweigert, hep-th/9905038.

[7] H. Garcia-Compean and J.F. Plebanski, hep-th/9907183.

[8] A. Alekseev, A. Recknagel and V. Schomerus, hep-th/9908040, JHEP 9909 (1999) 023.

[9] G. Felder, J. Frohlich, J. Fuchs and C. Schweigert, hep-th/9909030.

[10] J.M. Figueroa-O'Farrill and S. Stanciu, hep-th/0001199.

[11] C. Bachas, M. Douglas and C. Schweigert, hep-th/0003037.

[12] A. Alekseev, A. Recknagel and V. Schomerus, hep-th/0003187.

[13] S. Fredenhagen and V. Schomerus, hep-th/0012164.

[14] J. Maldacena, G. Moore and N. Seiberg, hep-th/0108100.

[15] K. Bardakci, E. Rabinovici and B. Saring, Nucl. Phys. B299 (1988) 151.

[16] K. Gawedzki and A. Kupiainen, Phys. Lett. B215 (1988) 119

[17] K. Gawedzki and A. Kupiainen, Nucl. Phys. B320 (1989) 625

[18] J. Maldacena, G. Moore and N. Seiberg, hep-th/0105038

[19] K. Gawedzki, hep-th/0108044.

[20] A. M. Polyakov and P. B. Wiegmann, Phys. Lett. 131B (1983) 121.

[21] A. M. Polyakov and P. B. Wiegmann, Phys. Lett. 141B (1984) 223.

[22] D. Karabali, Q-H Park, H. Schnitzer and Z. Yang, Phys. Lett. B216 (1989) 307.

[23] D. Karabali and H. Schnitzer, Nucl. Phys. B329 (1990) 649.

[24] P. Di Francesco, P. Mathieu and D. Senechal, Conformal Field Theory, 1997, SpringerVerlag, New York.

[25] D. Gepner, Phys. Lett. B222, (1989), 207.

[26] D. Gepner, Nucl. Phys. B322, (1989), 65. 